\title{
Desempenho produtivo e parâmetros fisiológicos de juvenis de pacu criados em tanques-rede em diferentes densidades de estocagem
}

\author{
Performance and physiological parameters of juvenile pacu reared in cages at different \\ stocking densities
}

\author{
MACIEL, Elayna Cristina da Silva ${ }^{1 *}$; FEITOSA, Kênia Cristine de Oliveira ${ }^{1}$; CORRÊA \\ NETO, Calix to Ramos ${ }^{1}$; MACEDO, Fernando Franceschini ${ }^{1}$; MATTIOLI, Wagner \\ Ormond $^{1}$; ABIMORAD, Eduardo Gianini ${ }^{2}$; ABREU, Janessa Sampaio de ${ }^{1}$
}

\footnotetext{
${ }^{1}$ Universidade Federal do Mato Grosso, Faculdade de Agronomia e Medicina Veterinária, Cuiabá, Mato Grosso, Brasil.

${ }^{2}$ Agência Paulista de Tecnologia dos Agronegócios, Pólo Regional do Noroeste Paulista, Votuporanga, São Paulo, Brasil.

*Endereço para correspondência: elaynamaciel@gmail.com
}

\section{RESUMO}

Objetivou-se investigar a influência da densidade de estocagem no desempenho zootécnico e parâmetros fisiológicos de juvenis de pacu (Piaractus mesopotamicus) criados em tanques-rede. Um total de 630 pacus $(56,81 \pm$ $7,76 \mathrm{~g}$ ) foi distribuído aleatoriamente em nove tanques-rede $\left(1 \mathrm{~m}^{3}\right)$. O delineamento foi inteiramente casualizado, com três densidades (30; 70 e 110 peixes $/ \mathrm{m}^{3}$ ) e três repetições. O experimento durou 90 dias com biometrias realizadas a cada 30, para mensuração do peso, comprimento total, taxa de crescimento específico e cálculo do fator de condição. Ao final dos 90 dias, os peixes foram submetidos à coleta de sangue para avaliação de glicemia, hematócrito, hemoglobina, proteína plasmática total e cloreto e foram retirados dos tanques-rede para a avaliação dos parâmetros de produtividade. Os resultados foram analisados por análise de regressão. Aos 30; 60 e 90 dias de criação, foi verificada efeito linear negativo para peso e comprimento, os quais diminuíram com o aumento das densidades de estocagem. Ao final dos 90 dias de criação, o ganho de peso diminuiu com o aumento das densidades e o inverso foi observado no ganho de biomassa. Não foi verificada diferenças para conversão alimentar aparente e taxa de sobrevivência. Somente concentração de hemoglobina, valor de hematócrito e níveis de proteína plasmática total se apresentaram com correlação linear positiva e houve aumento das densidades testadas. Apesar da disponibilidade restrita de espaço encontrada na maior densidade de estocagem ter exercido efeito adverso no crescimento dos pacus, esta foi considerada a mais adequada.

Palavras-chave: estresse, glicemia, peixe tropical, Piaractus mesopotamicus, produtividade.

\section{SUMARY}

This study investigated the influence of stocking density on the performance and physiological parameters of juvenile pacu (Piaractus mesopotamicus) reared in net cages. A total of 630 pacus $(56.81 \pm 7.76 \mathrm{~g})$ was randomly distributed into nine net cages $\left(1 \mathrm{~m}^{3}\right)$, located in pond without water renovation. The experimental design was completely randomized with three densities (30; 70 and $110 \mathrm{fish} / \mathrm{m}^{3}$ ) and three replicates. The experiment was conducted over 90 days, with biometric measurements carried out every 30 days. At the end of the experiment, blood samples were collected from the fish to evaluate blood glucose, hematocrit, hemoglobin concentration, total plasma protein and chloride and then they were removed from the net cages to evaluate the productivity parameters. The results were analyzed by regression analysis. At 30; 60 and 90 days, there was a linear effect for weight and length, which decreased with increasing stocking densities. At the end of 90 days, the weight gain decreased with increasing densities and the reverse was observed in 
biomass gain. There was no differences in feed conversion ratio and survival rate. Only hemoglobin concentration, hematocrit value and plasma total protein levels were presented with positive linear correlation and increased with increasing densities. In spite of limited space available in the highest density, there was no adverse effect on the growth of pacu raised in cages for 90 days and it was considered the most adequate since it was not limiting productivity by providing a state of comfort for fish, resulting in better production.

Keywords: blood glucose, freshwater fish, Piaractus mesopotamicus, production, stress.

\section{INTRODUÇÃO}

A piscicultura de água doce tem apresentado aumento progressivo em quase todo território nacional (BRASIL, 2010) e o uso de tanques-rede pode ser uma alternativa para se diversificar as técnicas de produção, por ter relativamente menor custo de implantação em relação à criação em viveiros escavados, além de dispensar o alagamento de novas áreas (ROTTA \& QUEIROZ, 2003).

Dentre as diversas regiões do país, a região Centro-Oeste, em especial o estado de Mato Grosso, conta com requisito ideais para produção de peixes em larga escala, sendo o cultivo de espécies nativas a principal atividade (BARROS et al., 2010). Os rios e reservatórios de usinas hidrelétricas do estado apresentam potencial para criação de peixes em tanques-rede, o que possibilita gerar emprego e renda, dando condições de sobrevivência à famílias ribeirinhas, atingidas por barragens.

A viabilidade do cultivo em tanquesrede reveste-se na produção de proteína animal em menor espaço, visando maior produtividade por área. Contudo, no desenvolvimento de um pacote de produção para uma espécie de peixe, o primeiro passo é a determinação da densidade de estocagem ideal, uma vez que baixas densidades de estocagem podem caracterizar aproveitamento deficiente da área disponível (GOMES et al., 2000), enquanto o adensamento pode provocar estresse nos peixes resultando em menor desempenho produtivo e maior susceptibilidade à doenças (BALDWIN, 2010).

$\mathrm{O}$ conhecimento e monitoramento da fisiologia dos peixes têm sido uma importante ferramenta na criação intensiva de diversas espécies de teleósteos, uma vez que as respostas biológicas ao estresse podem ser uma ferramenta útil para monitorar a saúde do ecossistema aquático, possibilitando a adoção de medidas preventivas e corretivas no sistema de produção (WEDEMEYER, 1996).

Dado o potencial zootécnico que possuem, estudos têm sido conduzidos com espécies nativas (VILELA \& HAYASHI, 2001; CAVERO et al., 2003; SOUZA-FILHO \& CERQUEIRA, 2003; BARCELLOS et al., 2004; BRANDÃO et al., 2005; OSTINI et al., 2007) a fim de definir parâmetros técnicos na criação em tanques-rede. $\mathrm{O}$ pacu (Piaractus mesopotamicus) tem grande importância na piscicultura brasileira (URBINATI et al., 2010) e é considerada uma das espécies mais apreciadas no Estado de Mato Grosso. No presente estudo, foi investigada a influência da densidade de estocagem no desempenho zootécnico e parâmetros fisiológicos de juvenis de pacu (Piaractus mesopotamicus) criados em tanques-rede de pequeno volume.

\section{MATERIAL E MÉTODOS}

O experimento foi realizado na Estação Experimental de Piscicultura da Faculdade de Agronomia, Medicina 
Veterinária e Zootecnia (FAMEVZ) da Universidade Federal de Mato Grosso (UFMT), Cuiabá, MT, no período de novembro de 2010 a janeiro de 2011. Foram utilizados 630 pacus (Piaractus mesopotamicus), obtidos pela Piscicultura Buriti (Nova Mutum/MT) com peso médio inicial de 56,81 $\pm 7,76 \mathrm{~g}$ distribuídos aleatoriamente em nove tanques-rede de $1 \mathrm{~m}^{3}$, confeccionados em multifilamento revestido com PVC (policloreto de venila), de malha de $10 \mathrm{~mm}$, alocados em um viveiro escavado de $800 \mathrm{~m}^{2}$ e $1,80 \mathrm{~m}$ de profundidade, sem renovação de água.

Os tratamentos experimentais foram constituídos de três diferentes densidades de estocagem: 30peixes $/ \mathrm{m}^{3} ; 70$ peixes $/ \mathrm{m}^{3}$ e 110 peixes $/ \mathrm{m}^{3}$, cada qual com três repetições. $\mathrm{O}$ experimento teve duração de 90 dias e, neste período, os peixes foram alimentados com ração comercial extrusada, $(36 \%$ de proteína bruta e $4 \mathrm{~mm}$ de diâmetro do pelet) duas vezes ao dia. A quantidade de ração fornecida diariamente foi calculada a partir de uma taxa de $5 \%$ sobre a biomassa de cada tanque-rede. A cada 30 dias foi realizada biometria, na qual $10 \%$ dos peixes de cada tratamento foram anestesiados (benzocaína, 66mg/L de água), pesados (balança Marte AS 2000C) e medidos através de ictiomêtro para cálculo do fator de condição de Fulton $\left(\mathrm{k}=\right.$ (peso/comprimento total $\left.{ }^{3}\right)$ *100), crescimento em peso e comprimento e taxa de crescimento específico $(\mathrm{TCE}=[(\ln$ pesotempo $0-\ln$ peso tempo 1)/tempo] x 100). Ao final do período experimental, os peixes foram mantidos em jejum por 24 horas e cinco peixes de cada tratamento foram amostrados ( $\mathrm{n}=5 /$ tratamento $)$, anestesiados (benzocaína, 66mg/L de água) e submetidos à coleta de sangue por punção da veia caudal, com seringas banhadas com anticoagulante EDTA. No sangue total foram avaliados a concentração de glicose (leitor digital AccuCheck-Active), hematócrito (Microcentrifuga modelo SPIN 1000, centrifugação por 5 minutos a $3300 \mathrm{x} \mathrm{g}$ ) e hemoglobina (Kit Labtest $\left.{ }^{\circledR}\right)$. O plasma resultante da centrifugação do hematócrito foi utilizado para avaliação de proteína plasmática total, através do método de refratometria. $\mathrm{O}$ sangue total foi centrifugado (centrífuga Edulab modelo 80-2B, centrifugação por 5 minutos a $3500 \mathrm{~g}$ ) e o plasma utilizado para análise de cloreto (Kit Labtest ${ }^{\circledR}$ ). No final do período experimental, os animais foram retirados dos tanquesrede para avaliação dos seguintes parâmetros de produtividade: conversão alimentar aparente (CAA) = consumo de ração $(\mathrm{kg}) /$ ganho de biomassa $(\mathrm{kg})$; ganho de peso $(\mathrm{GP})=$ peso médio final $(\mathrm{g})$ - peso médio inicial $(\mathrm{g})$; ganho de biomassa $(\mathrm{GB})=$ biomassa final $(\mathrm{kg})-$ biomassa inicial $(\mathrm{kg})$ e sobrevivência $(\%)$, avaliada pela diferença entre o número de peixes que iniciaram e que finalizaram o experimento em cada tratamento.

Durante todo o período experimental, amostras de água foram coletadas diariamente para análise de temperatura, oxigênio dissolvido (YSI 55, Yellow Springs Instruments) e $\mathrm{pH}$ (peagâmetro de bolso digital Quimis modelo Q400C), e, semanalmente, para avaliação de alcalinidade (a partir da solução indicadora de metil laranja), amônia total (kit comercial Alcon ${ }^{\mathbb{R}}$ ) e amônia não-ionizada, calculada segundo Emerson et al. (1975).

Utilizou-se o delineamento inteiramente casualizado, com três tratamentos e três repetições, sendo cada tanque-rede considerado como unidade experimental. As análises estatísticas das variáveis foram realizadas por análise de regressão, através do programa SAEG (UFV, 1982). A escolha dos modelos baseou-se na significância dos 
coeficientes por meio do teste $\mathrm{t}$ de Student a $5 \%$ de probabilidade

\section{RESULTADOS E DISCUSSÃO}

Os parâmetros físico-químicos da água (Tabela 1) apresentaram-se, em sua maioria, dentro do preconizado para a produção de peixes tropicais (PROENÇA \& BITTENCOURT, 1994). Neste trabalho, durante todo o período experimental não houve renovação de água no viveiro onde estavam instalados os tanques-rede, resultando em níveis de oxigênio dissolvido entre 3,81 a $3,89 \mathrm{mg} / \mathrm{L}$, abaixo do recomendado para maioria dos peixes, segundo Proença \& Bittencourt (1994). Devido à respiração branquial que os peixes apresentam, o oxigênio dissolvido na água é um fator limitante quando em baixas concentrações, prejudicando a sobrevivência. Contudo, estes baixos valores de oxigênio não foram prejudiciais aos pacus, uma vez que sobrevivências superiores a $98 \%$ foram registradas durante todo o período experimental.

Tabela 1. Parâmetros de qualidade da água durante 90 dias da criação de juvenis de pacu em tanques-rede em diferentes densidades de estocagem

\begin{tabular}{lrrr}
\hline \multirow{2}{*}{ Parâmetros } & \multicolumn{3}{c}{ Densidade de estocagem $\left(\right.$ peixes $\left./ \mathrm{m}^{3}\right)$} \\
\cline { 2 - 4 } & \multicolumn{1}{c}{30} & \multicolumn{1}{c}{70} & \multicolumn{1}{c}{110} \\
\hline Oxigênio dissolvido $(\mathrm{mg} / \mathrm{L})$ & $3,89 \pm 1,09$ & $3,88 \pm 1,13$ & $3,81 \pm 1,15$ \\
Temperatura $\left({ }^{\circ} \mathrm{C}\right)$ & $29,04 \pm 1,83$ & $29,06 \pm 1,83$ & $28,99 \pm 1,86$ \\
$\mathrm{pH}$ & $7,42 \pm 0,36$ & $7,44 \pm 0,33$ & $7,48 \pm 0,32$ \\
Alcalinidade total $(\mathrm{mg} \mathrm{CaCO} / \mathrm{L})$ & $58,12 \pm 11,89$ & $59,39 \pm 10,06$ & $61,75 \pm 11,88$ \\
Amônia Total $(\mathrm{mg} / \mathrm{L})$ & $0,67 \pm 1,00$ & $0,69 \pm 0,98$ & $0,86 \pm 1,07$ \\
Amônia não-ionizada $(\mathrm{mg} / \mathrm{L})$ & $0,003 \pm 0,005$ & $0,005 \pm 0,006$ & $0,005 \pm 0,006$ \\
\hline
\end{tabular}

Houve diferença significativa para peso e comprimento aos 30 (Figura 1 a e b), 60 (Figura 2 a e b) e 90 dias de criação (Figura 3 a e b), com correlação negativa para estes parâmetros de crescimento, os quais diminuíram com o aumento das densidades de estocagem. A taxa de crescimento específico diferiu significativamente somente aos 60 dias de criação, diminuindo à medida que aumentava a densidade de estocagem (Figura $2 \mathrm{c}$ ).

$\mathrm{Na}$ avaliação dos parâmetros de produtividade dos pacus (Piaractus mesopotamicus) ao final dos 90 dias de criação, a conversão alimentar aparente
(CAA) e taxa de sobrevivência não foram afetadas pelas densidades de estocagem testadas, uma vez que não foi observado efeito $(\mathrm{P}>0,05)$ para estes parâmetros. A conversão alimentar aparente obtida neste trabalho $(1,39$ a 1,63) foi inferior a 2,43-2,67, descrita por Bittencourt et al. (2010), para mesma espécie durante engorda em tanques-rede por 240 dias em diferentes densidades de estocagem $(40 ; 60$ e 80 peixes $/ \mathrm{m}^{3}$ ).

$\mathrm{O}$ ganho de peso diminuiu significativamente com o aumento das densidades (Figura 4a), (218,57 \pm $16,73 \mathrm{~g} ; 186,93 \pm 15,08 \mathrm{~g}$ e $170,99 \pm$ 
$28,27 \mathrm{~g}$, para densidade de 30; 70 e 110 peixes $/ \mathrm{m}^{3}$, respectivamente), o que está relacionado a maior disponibilidade de espaço encontrada em menores densidades. $\mathrm{O}$ inverso foi observado no ganho de biomassa (Figura 4b) que, ao final do experimento, apresentou-se maior na densidade de 110 peixes $/ \mathrm{m}^{3}$

a

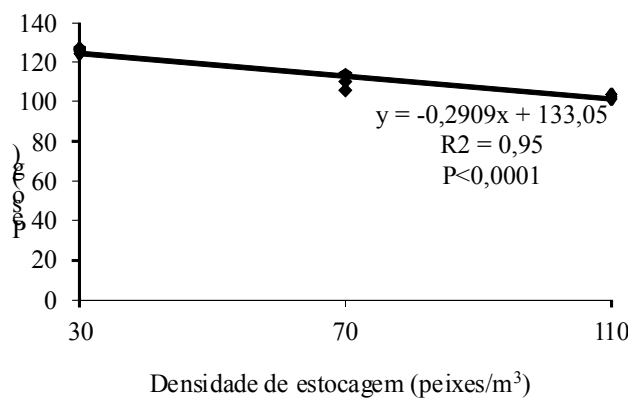

$(18,81 \mathrm{~kg})$ quando comparado às densidades de $70 \quad(12,68 \mathrm{~kg})$ e 30 peixes $/ \mathrm{m}^{3}(6,47 \mathrm{~kg})$. Isto é explicado pelo fato de que na maior densidade de estocagem, há um maior número de indivíduos, refletindo em aumento na biomassa total e consequentemente ganho de biomassa. b

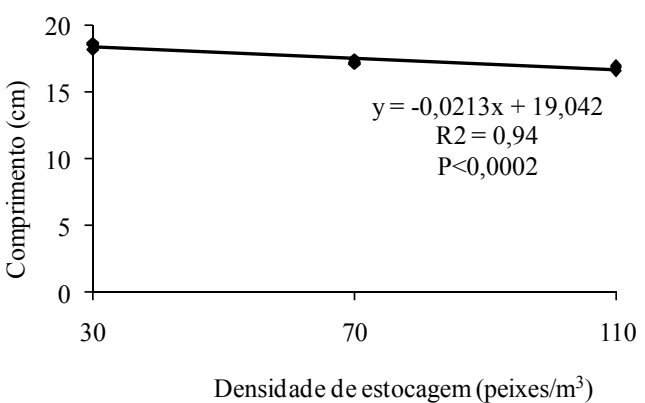

Figura 1. Peso (A) e comprimento (B) aos 30 dias de juvenis de pacu criados em tanques-rede em diferentes densidades de estocagem

a

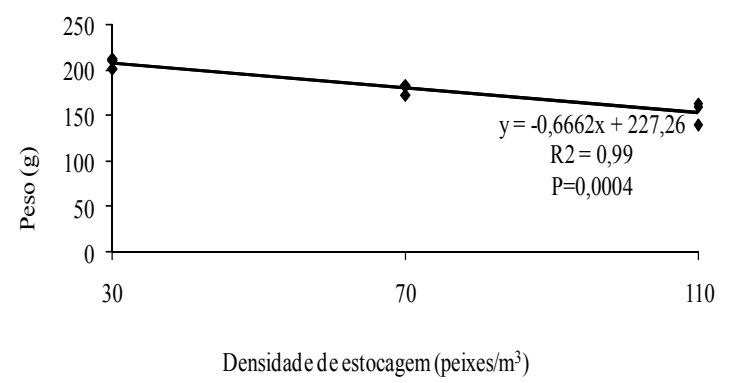

$\mathrm{b}$

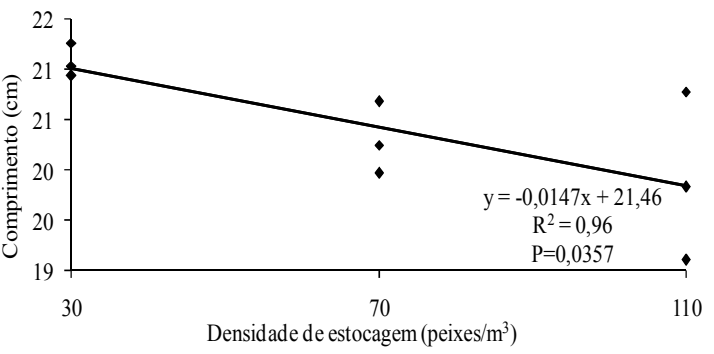

$\mathrm{c}$

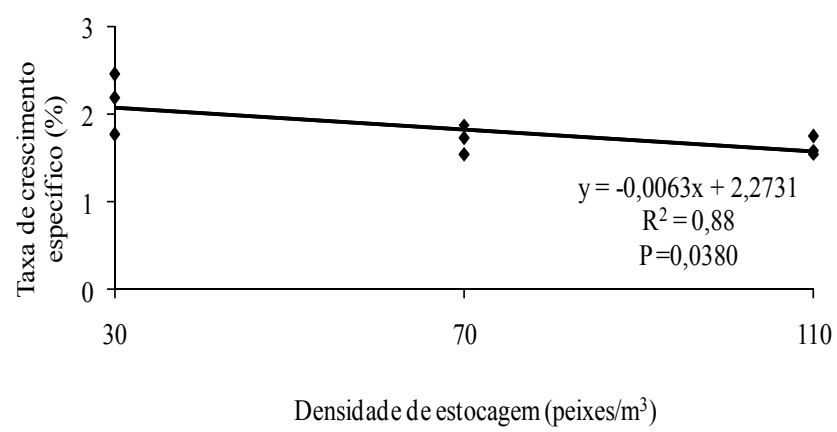

Figura 2. Peso (A), comprimento (B) e Taxa de Crescimento Específico (C) aos 60 dias de juvenis de pacu criados em tanques-rede em diferentes densidades de estocagem 
Rev. Bras. Saúde Prod. Anim., Salvador, v.14, n.1, p.185-194 jan./mar., 2013 http://www.rbspa.ufba.br ISSN 15199940

a

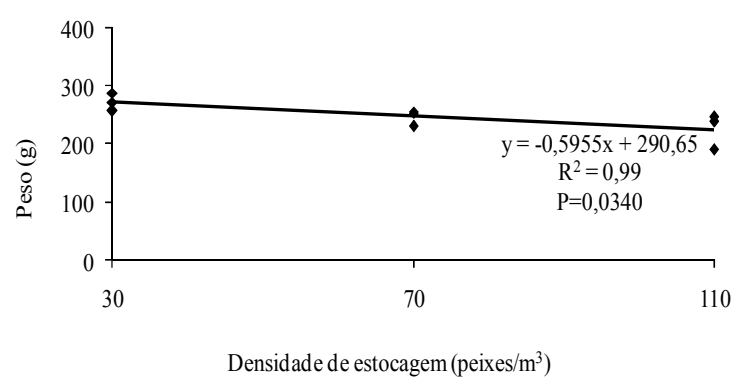

b

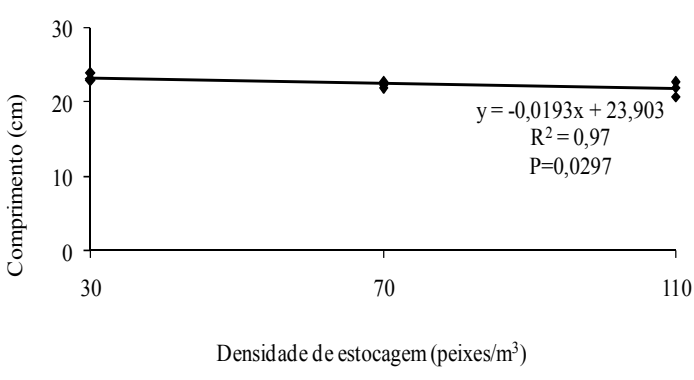

Figura 3. Peso (A) e comprimento (B) aos 90 dias de juvenis de pacu criados em tanques-rede em diferentes densidades de estocagem

a

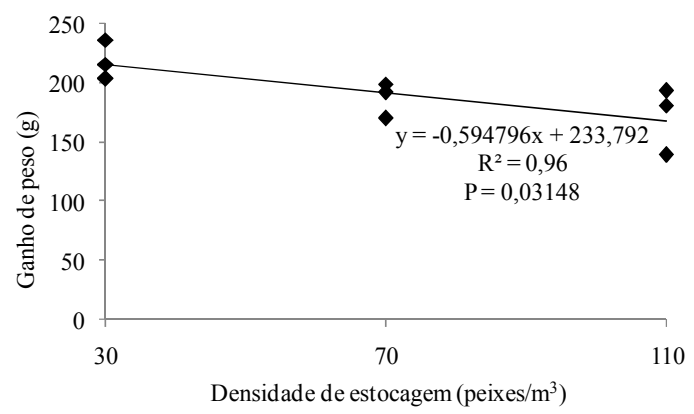

$\mathrm{b}$

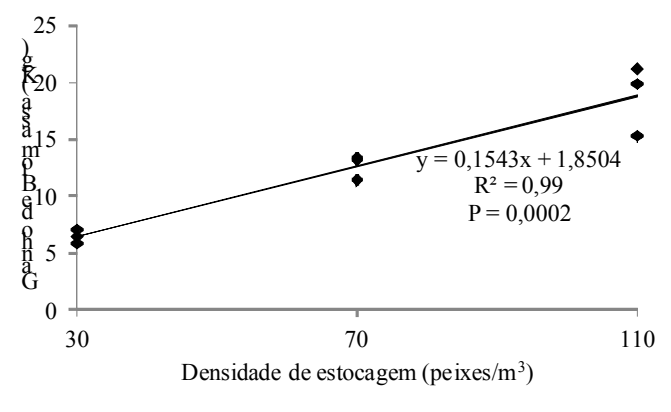

Figura 4. Ganho de peso (A) e ganho de biomassa (B) de juvenis de pacu criados em tanques-rede em diferentes densidades de estocagem por 90 dias

Resultados semelhantes foram observados em outras espécies de peixes. Brandão et al. (2004) verificaram, após 60 dias de criação de alevinos de tambaqui (Colossoma macropoтum) em tanques-rede, crescimento em comprimento e peso significativamente mais baixo na maior densidade $\left(500\right.$ peixes $\left./ \mathrm{m}^{3}\right)$ quando comparado a menor (200 peixes $\left./ \mathrm{m}^{3}\right)$. Similarmente, Bittencourt et al. (2010), trabalhando com densidades de estocagem de 40; 60 e 80 peixes $/ \mathrm{m}^{3}$, observaram que o aumento da densidade de estocagem proporcionou redução nos valores de peso final e ganho de peso em pacus após 240 dias de criação em tanques-rede. Já Carneiro et al. (1999), não verificaram diferença estatisticamente significativa entre os valores de peso médio e comprimento médio em tilápias vermelhas da Flórida criadas em tanques-rede por 253 dias nas densidades de 25; 50; 75 e 100 peixes $/ \mathrm{m}^{3}$ e Marques et al. (2004), testando em tanques-rede as densidades de 24, 48, 72 e 96peixes $/ \mathrm{m}^{3}$ para matrinxã (Brycon amazonicus), concluíram que as densidades não afetaram o ganho de peso $\mathrm{e}$ sobrevivência dos peixes. Em juvenis de dourado (Salminus brasiliensis), os índices de sobrevivência $(93,1 \%$ e $97,2 \%$ ) não foram afetados pelas diferentes densidades de estocagem (30, 150 e 300 peixes $\left./ \mathrm{m}^{3}\right)$, de acordo com Braun et al. 2009. Em Pangassus (Pangasius bocourti) não foi verificada diferenças significativas na conversão alimentar dos peixes criados nas 
densidades de 12; 25; 50; 100 e 200peixes $/ \mathrm{m}^{3}$ (JIWYAN, 2011), assim como em alevinos de surubim (Pseudoplatystoma spp), sendo que a densidade de estocagem (35; 70 e 110 peixes $/ \mathrm{m}^{3}$ ) em tanques-rede não afetou a conversão alimentar desta espécie (TURRA et al., 2009).

Em piscicultura, o aumento da produção pode ser alcançado, dentre outros fatores, pelo maior número de peixes por área cultivada. Contudo, a elevação das densidades aliadas às atividades rotineiras da criação podem ocasionar estresse e risco ao desempenho dos peixes (BRANDÃO et al., 2006). Em resposta a um estressor, os peixes desencadeiam uma série de mudanças bioquímicas e fisiológicas na tentativa de enfrentar o desafio imposto sobre eles, como alterações na glicemia, no número de células vermelhas e brancas e, ainda, nas concentrações plasmáticas de íons e proteínas (WENDELAAR BONGA, 1997). De acordo com Procarione et al. (1999), densidades de estocagem extremas causam um aumento na glicose sanguínea e consequente diminuição do crescimento, pois a energia destinada ao crescimento é desviada para compensar a situação desfavorável.

Estudos conduzidos por Brandão et al. (2006), mostraram que as práticas de manejo exercidas em pisciculturas, se não bem conduzidas, podem alterar os parâmetros fisiológicos dos peixes, e o adensamento aplicado em pirarucus (Arapaima gigas) provocou diferença significativa na taxa glicêmica $(99,5 \pm$ $3,2 \mathrm{mg} / \mathrm{dL}$ ) e nos valores de hematócrito 12 horas; 24 horas e 48 horas após adensamento. No presente trabalho, não foram observadas diferenças $(\mathrm{P}>0,05)$ para glicemia, cloreto e fator de condição (Figura 5).

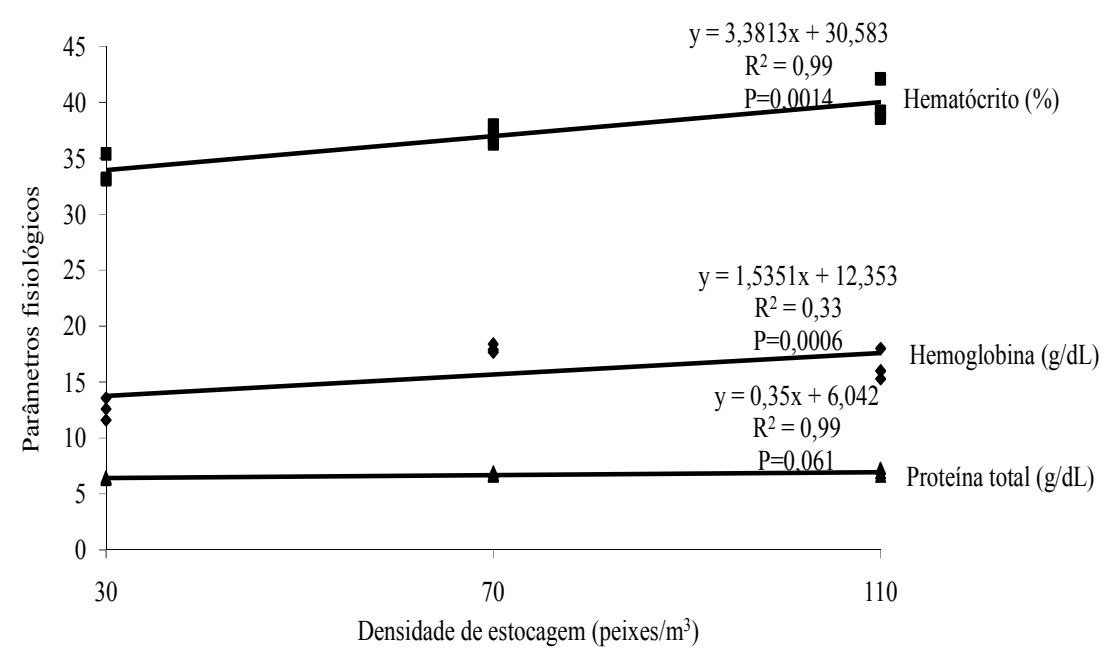

Figura 5. Hematócrito (\%), hemoglobina $(\mathrm{g} / \mathrm{dL})$ e proteína plasmática total $(\mathrm{g} / \mathrm{dL}) \mathrm{de}$ juvenis de pacu criados em tanques-rede em diferentes densidades de estocagem por 90 dias

Os demais parâmetros (concentração de hemoglobina, valor de hematócrito e níveis de proteína plasmática total) se apresentaram com correlação linear positiva $(\mathrm{P}<0,05)$ e aumentaram com aumento das densidades testadas (Figura 5). Apesar das diferenças significativas obtidas entre as 
Rev. Bras. Saúde Prod. Anim., Salvador, v.14, n.1, p.185-194 jan./mar., 2013 http://www.rbspa.ufba.br ISSN 15199940

densidades testadas, os valores encontrados neste trabalho para estes parâmetros são semelhantes aos valores basais, referenciados em outros trabalhos com pacus juvenis, quando não submetidos a nenhum distúrbio ou agente estressor (TAKAHASHI et al., 2006; ABREU et al., 2009; BITTENCOURT et al., 2010), evidenciando que nenhuma das densidades testadas foi fisiologicamente estressante para o pacu durante os 90 dias de criação.

Uma densidade de estocagem ótima é representada pela maior quantidade de peixes produzida eficientemente por unidade de volume de um tanque-rede. Produção eficiente não significa o peso máximo que pode ser produzido, mas sim o peso que pode ser produzido com uma conversão alimentar adequada, num período razoavelmente curto $\mathrm{e}$ com peso final aceito pelo mercado consumidor (SCHMITTOU, 1997). Com o aumento da densidade de estocagem, a biomassa total também aumenta, e esta relação direta entre densidade e produção de biomassa pode ser muito importante, até atingirse a biomassa econômica (KUBTIZA et al., 2004). No presente trabalho, apesar da disponibilidade restrita de espaço encontrada na maior densidade de estocagem $\left(110\right.$ peixes $\left./ \mathrm{m}^{3}\right)$ ter exercido efeito adverso no crescimento dos pacus criados em tanques-rede por 90 dias, ela não foi limitante à produtividade, proporcionando aos peixes um estado de conforto, o que resultou em melhor desempenho produtivo.

\section{AGRADECIMENTOS}

Os autores gostariam de agradecer à Fundação de Amparo à Pesquisa de Mato Grosso (FAPEMAT) pelo apoio financeiro, à Piscicultura Buriti pela doação dos peixes, à empresa Guabi pela doação da ração utilizada no experimento e ao Professor Joadil Gonçalves de Abreu (Universidade Federal de Mato Grosso) pelo auxílio nas análises estatísticas.

\section{REFERÊNCIAS}

ABREU, J.S.; TAKAHASHI, L.S.; HOSHIBA, M.A.; URBINATI, E.C. Biological indicators of stress in pacu (Piaractus mesopotamicus) after capture. Brazilian Journal of Biology, v.69, n.2, p.415-421, 2009.

BALDWIN, L. The effects of stocking density on fish welfare. The Plymouth Student Scientist, v.4, n.1, p.372-383, 2010.

BARCELLOS, L.J.G.; KREUTZ, L.C.; QUEVEDO, R.M.; FIOREZE, I.; CERICATO, L.; SOSO, A.B.; FAGUNDES, M.; CONRAD, J.; BALDISSERA, R.K.; BRUSCHI, A.; RITTER, F. Nursery rearing of jundiá Rhamdia quelen (Quoy \& Gaimard) in cages: cage type, stocking density and stress response to confinement.

Aquaculture, v.232, p.383-394, 2004.

BARROS, A.F; MARTINS, M.I.E.G.; ABREU, J.S.; AMARAL, C.M.C.

Investimento com implantação e custo de produção em piscicultura no Estado de Mato Grosso. Cáceres: UNEMAT, 2010. 87p.

BITTENCOURT, F.; FEIDEN, A.; SIGNOR, A.A.; BOSCOLO, W.R.; LORENZ, E.K; MALUF, M.L.F.

Densidade de estocagem e parâmetros eritrocitários de pacus criados em tanques-rede. Revista Brasileira de Zootecnia, v.39, n.11, p.2323-2329, 2010 . 
BRANDÃO, F.R.; GOMES, L.C.; CHAGAS, E.C.; ARAÚJO, L.D.

Densidade de estocagem de juvenis de tambaqui durante a recria em tanquesrede. Pesquisa Agropecuária

Brasileira, v.39, n.4, p.357-362, 2004.

BRANDÃO, F.R.; GOMES, L.C.; CHAGAS, E.C.; ARAÚJO, L.D.; SILVA, A.L.F. Densidade de estocagem de matrinxã (Brycon amazonicus) na recria em tanque-rede. Pesquisa

Agropecuária Brasileira, v.40, n.3, p.299-303, 2005.

BRANDÃO, F.R; GOMES, L.C.; CHAGAS, E.C. Respostas de estresses em pirarucu (Arapaima gigas) durante práticas de rotina em pisciculturas. Acta Amazônica, v.36, n.3 p.349-356, 2006.

BRASIL. Ministério da Pesca e Aquicultura. Produção Pesqueira e Aquícola- estatística. 2010. Disponível em: <http://www.mpa.gov.br>. Acesso em: 16 jul. 2011.

BRAUN, N.; LIMA, L.R.; BALDISSEROTTO, B. Growth, biochemical and physiological responses of Salminus brasiliensis with different stocking densities and handling.

Aquaculture, v.301, p.22-30, 2009.

CARNEIRO, P.C.F.; CYRINO, J.E.P.; CASTAGNOLLI, N. Produção de tilápia vermelha da Flórida em tanques-rede.

Scientia Agricola, v.56, n.3, p.673-679, 1999.

CAVERO, B.A.A.; PEREIRA-FILHO, M.; ROUBACH, R.; ITUASSÚ, D.R.; GANDRA, A.L.; CRESCÊNCIO, R. Efeito da densidade de estocagem sobre a eficiência alimentar de juvenis de Pirarucu (Arapaima gigas) em ambiente confinado. Acta Amazonica, v.33, n.4, p.631-636, 2003.
EMERSON, K.; RUSSO, R.C.; LUND, R.E.; THURSTON, R.V. Aqueous ammonia equilibrium calculations: effects of $\mathrm{pH}$ and temperature. Journal of the Fisheries Research Board of Canada, v.32, p.2379-2383, 1975.

GOMES, L.C.; BALDISSEROTTO, B.; SENHORINI, J.A. Effect of stocking density on water quality, survival, and growth of larvae of matrinxã, Brycon cephalus (Characidae), in ponds. Aquaculture, v.183, p.73-81, 2000.

JIWYAN, W. The effect of stocking density on yield, growth and survival of Asian river catfish (Pangasius bocourti Sauvage, 1880). Aquaculture International, 2011. Disponível em: $<$ http://www.springerlink.com/content/ k531q58584654438/fulltext.htmi>. Acesso em: 23 ago.2011.

KUBITZA, F.; LOVSHIN, L.; ONO, A.E.; SAMPAIO, V.A. Planejamento da Produção de peixes. 4.ed. Jundiaí: F. Kubitza, 2004. 58p.

MARQUES, R.N.; HAYASHI, C.; FURUYA, M.W. Influência da densidade de estocagem no cultivo de alevinos de matrinxã Brycon cephalus (Günther,1869) em condições experimentais. Biological Sciences, v.26, n.1, p.55-59, 2004.

OSTINI, S.; OLIVEIRA, I.R.; SERRALHEIRO, P.C.S.; SANCHES, E.G. Criação do robalo-peva (Centropomus parallelus) submetido a diferentes densidades de estocagem.

Revista Brasileira de Saúde e Produção Animal [Online], v.8, n.3, p.250-257, 2007. 
PROENÇA, C.E.M; BITTENCOURT, P.R.L. Manual de piscicultura tropical. Brasília: IBAMA, 1994. 196p.

PROCARIONE, L.S.; BARRY, T.P.; MALISON, J.A. Effects of high rearing density and loading rates on the growth and stress responses of juvenile rainbow trout. North American Journal of Aquaculture, v.61, p.9196, 1999.

ROTTA, M.A.; QUEIROZ, J.F. Boas práticas de manejo (BPMs) para a produção de peixes em tanques-rede. Corumbá: EMBRAPA Pantanal, 2003. $27 \mathrm{p}$.

SCHMITTOU, H.R. Produção de peixes em alta densidade em tanquesrede de pequeno volume. Campinas:

Silvio Romero Coelho/Mogiana Alimentos S.A./ASA, 1997. 78f.

SOUZA-FILHO, J.J.; CERQUEIRA, V.R. Influência da densidade de estocagem no cultivo de juvenis de robalo-flecha mantidos em laboratório.

Pesquisa Agropecuária Brasileira, v.38, n.11, p.1317-1322, 2003.

TAKAHASHI, L.S.; ABREU, J.S.; BILLER., J.D.; URBINATI, E.C. Efeito do ambiente pós-transporte na recuperação dos indicadores de estresse de pacus juvenis (Piaractus mesopotamicus). Acta Scientiarum.

Animal Sciences, v.28, n.4, p.469-475, 2006.
TURRA, E.M; QUEIROZ, B.M; TEIXEIRA, E.A; FARIA, P.M.C.; CREPALDI, D.V.; RIBEIRO, L.P. Densidade de estocagem do surubim Pseudoplatystoma spp cultivado em tanque-rede. Revista Brasileira de Produção Animal, v.10, n.1, p.177187, 2009.

UNIVERSIDADE FEDERAL DE VIÇOSA - UFV. SAEG. Sistema para análise estatística e genética. Viçosa, 1982. 59p.

URBINATI, E.C.; GONCALVES, F.D.; TAKAHASHI, L.S. Pacu

(Piaractus mesopotamicus). In: BALSISSEROTO, B.; GOMES, L.C. Espécies nativas para piscicultura no Brasil. 2.ed. Santa Maria: Universidade Federal de Santa Maria, 2010. p.205244.

VILELA, C.; HAYASHI, C.

Desenvolvimento de juvenis de lambari Astyanax bimaculatus (Linnaeus, 1758), sob diferentes densidades de estocagem em tanques-rede. Acta Scientiarum, v.23, n.2, p.491-496, 2001.

WEDEMEYER, G.A. Physiology of fish in intensive culture systems. London: Chapman \& Hall, 1996. p.1059.

WENDELAAR BONGA, S.E. The stress response in fish. Physiological Reviews, v.77, n.3, p.591-625, 1997.

Data de recebimento: 13/12/2011

Data de aprovação: 17/12/2012 